\title{
Effects of extended powered knee prosthesis stance time via visual feedback on gait symmetry of individuals with unilateral amputation: a preliminary study
}

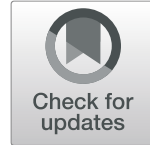

Andrea Brandt ${ }^{1,2}$, William Riddick ${ }^{3}$, Jonathan Stallrich ${ }^{4}$, Michael Lewek ${ }^{5}$ and He Helen Huang ${ }^{1,2^{*}}$

\begin{abstract}
Background: Establishing gait symmetry is a major aim of amputee rehabilitation and may be more attainable with powered prostheses. Though, based on previous work, we postulate that users transfer a previously-learned motor pattern across devices, limiting the functionality of more advanced prostheses. The objective of this study was to preliminarily investigate the effect of increased stance time via visual feedback on amputees' gait symmetry using powered and passive knee prostheses.
\end{abstract}

Methods: Five individuals with transfemoral amputation or knee disarticulation walked at their self-selected speed on a treadmill. Visual feedback was used to promote an increase in the amputated-limb stance time. Individuals were fit with a commercially-available powered prosthesis by a certified prosthetist and practiced walking during a prior visit. The same protocol was completed with a passive knee and powered knee prosthesis on separate days. We used repeated-measures, two-way ANOVA $(a l p h a=0.05)$ to test for significant effects of the feedback and device factors. Our main outcome measures were stance time asymmetry, peak anterior-posterior ground reaction forces, and peak anterior propulsion asymmetry.

Results: Increasing the amputated-limb stance time via visual feedback significantly improved the stance time symmetry $(p=0.012)$ and peak propulsion symmetry $(p=0.036)$ of individuals walking with both prostheses. With the powered knee prosthesis, the highest feedback target elicited 36\% improvement in stance time symmetry, 22\% increase in prosthesis-side peak propulsion, and $47 \%$ improvement in peak propulsion symmetry compared to a no feedback condition. The changes with feedback were not different with the passive prosthesis, and the main effects of device/ prosthesis type were not statistically different. However, subject by device interactions were significant, indicating individuals did not respond consistently with each device (e.g. prosthesis-side propulsion remained comparable to or was greater with the powered versus passive prosthesis for different subjects). Overall, prosthesisside peak propulsion averaged across conditions was 31\% greater with the powered prosthesis and peak propulsion asymmetry improved by $48 \%$ with the powered prosthesis.

(Continued on next page)

\footnotetext{
*Correspondence: helen-huang@unc.edu

This study was performed at North Carolina State University, Raleigh, NC 27606.

'Joint Department of Biomedical Engineering, North Carolina State

University, 4402D Engineering Building III, NC State University, Raleigh, NC 27606, USA

${ }^{2}$ The University of North Carolina at Chapel Hill, Chapel Hill, NC 27599, USA

Full list of author information is available at the end of the article
}

(c) The Author(s). 2019 Open Access This article is distributed under the terms of the Creative Commons Attribution 4.0 International License (http://creativecommons.org/licenses/by/4.0/), which permits unrestricted use, distribution, and reproduction in any medium, provided you give appropriate credit to the original author(s) and the source, provide a link to the Creative Commons license, and indicate if changes were made. The Creative Commons Public Domain Dedication waiver (http://creativecommons.org/publicdomain/zero/1.0/) applies to the data made available in this article, unless otherwise stated. 
(Continued from previous page)

Conclusions: Increasing prosthesis-side stance time via visual feedback favorably improved individuals' temporal and propulsive symmetry. The powered prosthesis commonly enabled greater propulsion, but individuals adapted to each device with varying behavior, requiring further investigation.

Keywords: Gait; Amputation; Visual Feedback; Rehabilitation; Knee Prosthesis,

\section{Introduction}

Establishing gait symmetry is a major aim of amputee rehabilitation to reduce the risk of secondary complications that often accompany asymmetric gait (e.g. back pain, osteoarthritis) [1-4]. Individuals with transfemoral amputation commonly exhibit reduced stance time and ground reaction forces from the prosthetic limb compared to their intact limb [5-8], coinciding with increased prevalence of secondary complications relative to individuals with more distal levels of amputation [3, 4]. Reduced propulsion from one limb has been associated with increased energy cost of walking $[9,10]$. Yet, prior work has suggested asymmetry is a functional result of the biomechanical constraints of energeticallypassive prostheses that need not be corrected [11-13]. Of note, passive prostheses cannot provide the necessary levels of joint torque and power during walking. The advent and commercialization of powered prostheses capable of mimicking the force generating behavior around biological joints [14-16] provides reason to revisit this perspective on gait symmetry.

Powered knee prostheses reduce biomechanical constraints by partially replacing the function of lower-limb biological muscles. To match the user, however, powered prostheses need to be set specifically for each individual. This personalization of powered knee control parameters can influence users' gait symmetry [17], and the ability of a powered prosthesis to provide net power leads us to believe these devices can reduce propulsive and temporal asymmetry; however, amputees typically spend less time on the powered prosthesis compared to the intact side, even with personalized control parameters [17-19]. Because amputees appear to maintain temporal walking strategies across devices [20], this asymmetric behavior may transfer to powered devices as well. We postulate that this transfer of a previouslylearned maladaptive motor pattern limits the functionality of more advanced prostheses.

Previously, real-time visual feedback has induced immediate improvements in temporal, spatial, and force symmetry for amputees walking with passive prostheses [21], and a case report demonstrated kinematic and metabolic improvements with such training [22]. To our knowledge, no one has investigated the ability and associated effects of amputees walking with a powered knee prosthesis to modulate their gait in response to visual feedback. This knowledge may improve amputees' gait asymmetries that persist in spite of technological advancements and broaden the number of amputees that are candidates for powered prostheses.

The objective of this study was to preliminarily investigate the effects of increasing stance time on a commercially-available powered knee prosthesis via visual feedback on amputees' gait symmetry without a walking aid. The ability to attain and maintain complete symmetry may be unrealistic given the biomechanical constraints that persist with powered prostheses (e.g. limited ankle motion), so our paradigm simply encourages a greater level of symmetry via increased stance time on the prosthetic limb. We hypothesized that with visual feedback targeting longer stance times, amputees would walk with improved temporal and force symmetry and reduced prosthesis-side hip power due to increased propulsion from the prosthesis. Second, we hypothesized that temporal measures may be similar between devices due to the transfer of similar motor patterns, but the powered prosthesis would enable greater improvements in propulsion, hip power, and perceived difficulty, considering its functional advantages.

\section{Methods}

\section{Participants and experimental setup}

We initially screened 11 people and recruited five people to participate in this study. All participants reported daily prosthetic wear for 12 or more hours and experience with treadmill walking (Table 1). Participants were excluded if they exhibited body weight/limb clearance not meeting Power Knee ${ }^{\mathrm{Tw}}$ (Össur; Reykjavik, Iceland) limitations and vision/cardiovascular complications that may affect his/her ability to complete the protocol. All subjects provided informed, written consent to participate in our protocol approved by the Institutional Review Board of the University of North Carolina at Chapel Hill.

The passive prostheses used in this study were Subjects 1-4's prescribed, microprocessor-controlled, energetically-passive knee prostheses (Table 1). The commercially-available powered knee prosthesis used in this study was the Power Knee ${ }^{\text {rm }}$ (Össur; Reykjavik, Iceland). Subject 5 used this powered prosthesis daily and did not have a passive prosthesis for comparison. A certified prosthetist, trained in alignment and fitting of 
Table 1 Participant information

\begin{tabular}{|c|c|c|c|c|c|}
\hline Subject & 1 & 2 & 3 & 4 & 5 \\
\hline Gender & Male & Male & Male & Male & Female \\
\hline Height & $1.8 \mathrm{~m}$ & $1.8 \mathrm{~m}$ & $1.7 \mathrm{~m}$ & $1.8 \mathrm{~m}$ & $1.7 \mathrm{~m}$ \\
\hline Body Weight & $69 \mathrm{~kg}$ & $94 \mathrm{~kg}$ & $61 \mathrm{~kg}$ & $69 \mathrm{~kg}$ & $49 \mathrm{~kg}$ \\
\hline Age & 24 years & 59 years & 27 years & 19 years & 52 years \\
\hline Time since Amputation & 7 years & 47 years & 4 years & 19 years & 27 years \\
\hline Reason for Amputation & Cancer & Cancer & Trauma & Congenital & Trauma \\
\hline Side of Amputation & $\begin{array}{l}\text { Right transfemoral } \\
\text { amputation }\end{array}$ & $\begin{array}{l}\text { Left transfemoral } \\
\text { amputation }\end{array}$ & Right knee disarticulation & $\begin{array}{l}\text { Left transfemoral } \\
\text { amputation }\end{array}$ & $\begin{array}{l}\text { Right transfemoral } \\
\text { amputation }\end{array}$ \\
\hline Prescribed Prosthesis & $\begin{array}{l}\text { Genium (Ottobock, } \\
1.7 \mathrm{~kg} \text { ) }\end{array}$ & $\begin{array}{l}\text { Genium (Ottobock, } \\
1.7 \mathrm{~kg} \text { ) }\end{array}$ & $\begin{array}{l}\text { Plie } 3 \text { (Freedom Innovations, } \\
1.2 \mathrm{~kg} \text { ) }\end{array}$ & $\begin{array}{l}\text { Rheo Knee (Ossur, } \\
1.6 \mathrm{~kg} \text { ) }\end{array}$ & $\begin{array}{l}\text { Power Knee } \\
\text { (Ossur, } 3.2 \mathrm{~kg} \text { ) }\end{array}$ \\
\hline $\begin{array}{l}\text { Time with Current } \\
\text { Prosthesis }\end{array}$ & 6 years & 3 years & 3 years & 6 months & 5 years \\
\hline $\begin{array}{l}\text { Physical Therapy Post- } \\
\text { amputation }\end{array}$ & 2 months & 6 months & 9 months & None & 3 or more months \\
\hline Suspension & $\begin{array}{l}\text { Double-wall with pin } \\
\text { to outer socket }\end{array}$ & $\begin{array}{l}\text { Ischial containment } \\
\text { suction }\end{array}$ & $\begin{array}{l}\text { Shuttle lock, 3-5-ply socks, } \\
\text { window socket with lanyard }\end{array}$ & Suction & Vacuum \\
\hline $\begin{array}{l}\text { Self-reported Functional K- } \\
\text { level }\end{array}$ & 3 & 3 & 3 & 4 & 3 \\
\hline $\begin{array}{l}\text { Self-selected Walking Speed } \\
\text { with Each Prosthesis }\end{array}$ & $\begin{array}{l}\text { Prescribed: } 0.8 \mathrm{~m} / \mathrm{s} \\
\text { Powered: } 0.8 \mathrm{~m} / \mathrm{s}\end{array}$ & $\begin{array}{l}\text { Prescribed: } 0.8 \mathrm{~m} / \mathrm{s} \\
\text { Powered: } 0.8 \mathrm{~m} / \mathrm{s}\end{array}$ & $\begin{array}{l}\text { Prescribed: } 0.9 \mathrm{~m} / \mathrm{s} \text { Powered: } \\
0.7 \mathrm{~m} / \mathrm{s}\end{array}$ & $\begin{array}{l}\text { Prescribed: } 0.5 \mathrm{~m} / \mathrm{s} \\
\text { Powered: } 0.7 \mathrm{~m} / \mathrm{s}\end{array}$ & $\begin{array}{l}\text { Powered (i.e. } \\
\text { prescribed): } 0.5 \mathrm{~m} / \\
\mathrm{s}\end{array}$ \\
\hline
\end{tabular}

The listed prescribed prosthesis for Subjects 1-4 are energetically-passive knee prostheses. Subject 5 wore a powered prosthesis daily and was only tested with the powered prosthesis

the powered prosthesis, aligned and tuned the powered prosthesis for Subjects 1-4 (Table 2). Subject 5 used her daily settings. Due to his long residual limb, Subject 3 wore a low-profile high-performance foot similar to his prescribed foot, so that he did not need a lift on his intact foot to obtain proper alignment. Subject 5 wore her prescribed foot (Proflex; Össur; Reykjavik, Iceland)), and the remaining subjects wore the ProFlex XC (Össur; Reykjavik, Iceland) with the powered knee (for optimal performance of the powered knee) and their prescribed foot with their prescribed knee. Each subject maintained his/her same socket, suspension, and shoes across all testing conditions to reduce confounding effects.

\section{Visual feedback}

Due to the high variability in amputees' spatial symmetry [23] and observed difficulty of inter-limb symmetry measures [21], we chose a unilateral, temporal metric (i.e. prosthetic-limb stance time) for visual feedback. More complex measures may also require longer training periods and the assistance of a physical therapist [24], limiting the accessibility of the intervention.

We created custom code for the real-time visual feedback display using Vicon DataStream SDK (VICON; Oxford, UK) and MATLAB (The MathWorks, Inc.; Natick, Massachusetts, USA) and displayed it on a $0.6-\mathrm{m}$ computer monitor at eye-level, $1 \mathrm{~m}$ in front of the treadmill (Fig. 1). We calculated prosthetic-limb stance time as the amount of time we detected a vertical ground reaction force greater than $10 \%$ of the subject's body weight, averaged it over the previous five strides [25] to reduce subjects' tendency to generate large stride-to-stride corrections, and updated it after each ipsilateral toe-off event (Fig. 1, blue dot). The subject's preferred stance time on each limb was extracted during the first trial, a no-feedback (None) trial, and used to set three visual feedback targets. Level 1 (L1) corresponded with the typical stance time of the prosthetic-limb, Level 3 (L3)

Table 2 Commercially-available powered knee prosthesis settings

\begin{tabular}{|c|c|c|c|c|c|c|}
\hline & Thigh length & Knee center height & Foot size & Pre-swing thigh angle & Maximum flexion angle & Swing initiation angle \\
\hline 1 & $48 \mathrm{~cm}$ & $50 \mathrm{~cm}$ & $26 \mathrm{~cm}$ & 20 & 60 & 15 \\
\hline 2 & $42 \mathrm{~cm}$ & $55 \mathrm{~cm}$ & $26 \mathrm{~cm}$ & 30 & 60 & 10 \\
\hline 3 & $54 \mathrm{~cm}$ & $35 \mathrm{~cm}$ & $26 \mathrm{~cm}$ & 20 & 60 & 15 \\
\hline 4 & $55 \mathrm{~cm}$ & $46 \mathrm{~cm}$ & $26 \mathrm{~cm}$ & 28 & 60 & 20 \\
\hline 5 & $43 \mathrm{~cm}$ & $53 \mathrm{~cm}$ & $26 \mathrm{~cm}$ & \multicolumn{3}{|c|}{ Unknown; set by her own prosthetist } \\
\hline
\end{tabular}




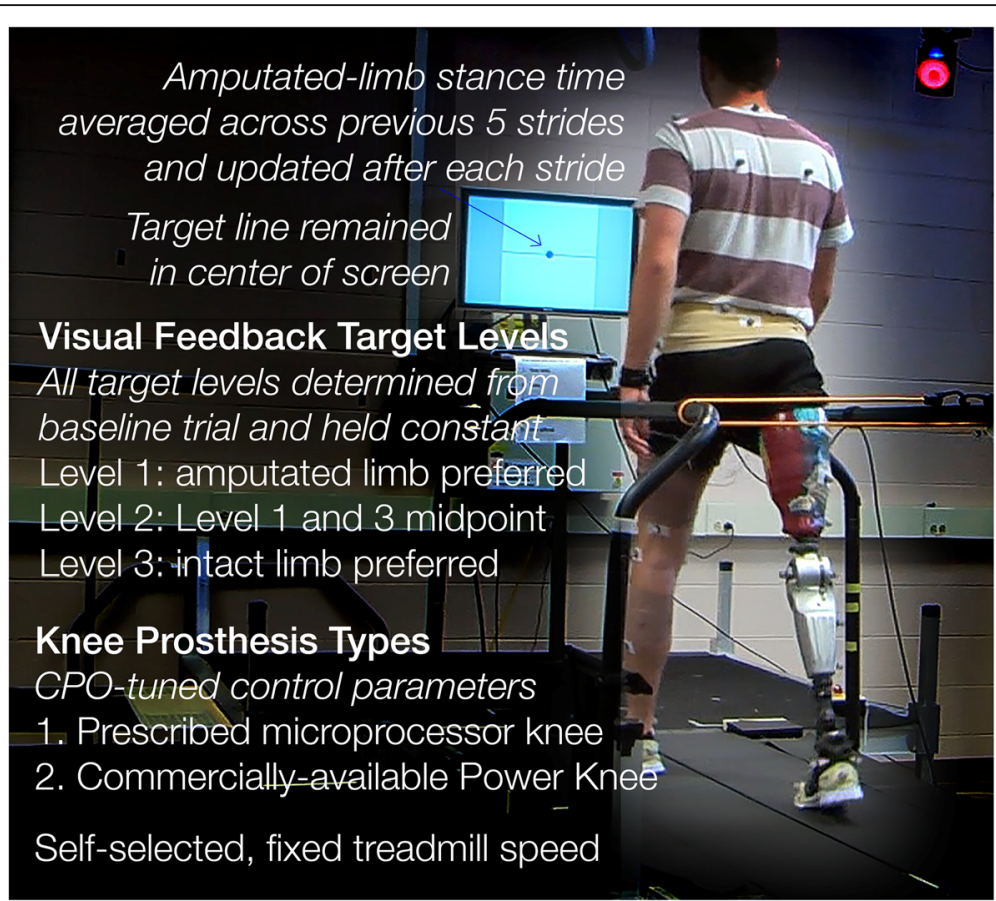

Fig. 1 Experimental design and visual feedback display. We asked each subject to walk at their self-selected treadmill speed with their prescribed passive knee prosthesis and the commercially-available powered knee prosthesis on two different testing days. On each day, their speed was held constant as they completed 3 replicate trials of each visual feedback target level and a no-feedback condition (total 12 trials). The blue dot in the center of the display, representing the stance time of the amputated-side (in seconds) averaged across the previous 5 strides, updated after every stride by moving up and down the y-axis

corresponded with the stance time of the intact-limb, and Level 2 (L2) was set at the midpoint between L1 and L3. The targets remained constant to encourage consistency in walking patterns and were centered on the screen to blind the subject from which level they were targeting (Fig. 1). The display range remained $+/-$ $0.2 \mathrm{~s}$ from the target line to maintain subjects' perceived accuracy.

\section{Experimental design}

Subjects visited the lab for a total of 3 days for powered knee prosthesis fitting and training with the prosthetist, testing with the prescribed (i.e. passive) knee prosthesis, and testing with the powered knee prosthesis. Each visit typically lasted $3 \mathrm{~h}$. Subjects performed the prescribed prosthesis testing first to ensure familiarity with the protocol prior to introducing a new device. On the fitting and training day, the prosthetist aligned and tuned the control parameters of the powered prosthesis (Table 2), and subjects practiced walking with the powered prosthesis until they felt comfortable on the treadmill without assistance.

At the beginning of each testing day, we determined the subject's self-selected walking speed on the treadmill (Table 1) and familiarized him/her with the Perceived Difficulty scale and visual feedback paradigm. To determine the self-selected speed, we started at a comfortable walking speed, increased/decreased the speed by $0.1 \mathrm{~m} / \mathrm{s}$ increments until the subject stated that the speed was too fast/slow, repeated this increase/decrease procedure three times in each direction while recording the speed that was noted as too fast/slow, and averaged these six speeds for their final self-selected speed (modified from [26]). Prior to testing, we provided time for subjects to practice using the visual feedback, but we kept this time minimal (until the subject confirmed they were comfortable, usually $30 \mathrm{~s}$ ) to prevent fatigue.

During testing, subjects completed a total of twelve 1.5-min walking trials with two or more minutes of rest between trials. We randomized the four conditions (i.e. None, L1-3) within three testing periods to prevent training time or fatigue from confounding our results. The first trial was None, in order to determine L1-3.

We recorded the ground reaction forces of each limb from a dual-belt treadmill $(1000 \mathrm{~Hz}$; Bertec Corp.; Columbus, OH, USA) and full-body motion using a 12-camera motion capture system $(100 \mathrm{~Hz}$; VICON; Oxford, UK). After each trial, we recorded subjects' Perceived Difficulty using a 9-item Likert scale: 1 corresponded to "very easy", and 9 corresponded to "very difficult".

To evaluate amputees' ability and strategy to modulate their walking pattern, we quantified their target-hitting 
accuracy and temporal gait measures (i.e. stance time, swing time, stance time asymmetry). To evaluate the associated effects on subjects' locomotor function and proximal joints, we quantified peak anterior propulsive force (and asymmetry), posterior braking force, and hip joint power of each limb [27]. Due to the large variability between amputee subjects, we felt the need to use primarily global metrics (interlimb symmetry and perceived difficulty), and the hip is the only intact joint with which we can observe interlimb symmetry.

\section{Data processing}

We selected nine consecutive strides from each trial, as this was the maximum number of consecutive strides in which subjects did not scuff the treadmill or touch the handrails across trials. For subject 5 only, we were unable to extract nine clean, consecutive strides from three trials (i.e. two None trials and one L1 trial), so we excluded these trials. For the visual feedback trials, we selected consecutive strides in which the subject was most consistently hitting the target. We low-pass filtered (Butterworth, 4th order, $7.5 \mathrm{~Hz}$ ) the ground reaction force and motion data, baseline-corrected the ground reaction force data, and used a $20 \mathrm{~N}$ threshold to identify gait events. We calculated sagittal-plane hip joint kinetics after manually adjusting the inertial properties of the prosthesis segments (Visual 3D; C-Motion, Inc.; Germantown, MD, USA). We normalized ground reaction forces and hip power to body mass and identified peak braking/propulsive forces from the anterior-posterior ground reaction force. For peak hip power, we identified maximum hip power during the second power generating interval of the stance phase, as it aligns with anterior propulsion. To evaluate inter-limb symmetry, we used a standard asymmetry index (ASI) $[7,28]$ :

$$
A S I=\frac{x_{i}-x_{p}}{\left(x_{i}+x_{p}\right) * 0.5} * 100,
$$

where $x_{i}$ and $x_{p}$ are the outcome measure for each stride of the intact and prosthesis side, respectively. Target-hitting accuracy was calculated as the absolute value of the error between subjects' prosthesis-side stance time and the target value for each stride using the same $10 \%$ body weight threshold.

\section{Statistical tests}

We averaged the 3 replicate trials for each testing condition and used repeated-measures, two-way ANOVA (alpha $=0.05)$ to test for significant effects of the feedback and device (i.e. knee prosthesis type) factors (JMP; SAS Institute; Cary, NC, USA). The analysis included random subject effects and their interactions with feedback and device, and fixed effects for testing period. The model R-squared value exceeded 0.90 for our main response variables. We used the Shapiro-Wilk normality test $(p<0.01)$ to detect outlier trials (Table 3$)$. When we found a significant main effect, we used Tukey's honestly significant difference test (alpha $=0.05$ ) to test for statistical difference between conditions. To investigate subject/device variance, we included within-subject comparisons for the kinetic measures (alpha $=0.05$ ) (Fig. $4)$. We reported effect sizes as $\eta_{p}^{2}\left(\mathrm{SS}_{\text {effect }} /\left(\mathrm{SS}_{\text {effect }}+\mathrm{SS}_{\text {ef- }}\right.\right.$ fect"subject)), statistical power as $1-\beta$, and mean and standard deviations throughout. Subject 5 did not have a passive prosthesis, so we did not include her in the statistical analysis to maintain equal sample sizes.

\section{Results}

Subjects' accuracy in hitting each target did not differ significantly between device (i.e. knee prosthesis type) or feedback conditions, but perceived difficulty significantly increased with visual feedback level (Table 4). Subject 5 had an average accuracy of $0.08 \mathrm{~s}$ (6\% of stride time) with the powered prosthesis, and her perceived difficulty followed the same trend with an average score of 2, 5, 5, and 6 (None to L3).

With increasing levels of visual feedback (i.e. None/L1 to L3), stance time on both limbs significantly increased (prosthesis-side $p=0.002, \quad \eta_{p}^{2}=0.79$; intact-side $p=$ $\left.0.005, \eta_{p}^{2}=0.74\right)$, and stance time symmetry significantly improved ( $p=0.012, \eta_{p}^{2}=0.69$ ) (Fig. 2). L3 significantly differed from None (and L1) conditions with $9 \%$ greater stance time on the prosthesis side, $4 \%$ greater stance time on the intact side, and 36\% improved stance time symmetry with the powered prosthesis. Device main effects were not significant (prosthesis-side $p=0.856,1$ $\beta=0.96$; intact-side $p=0.335,1-\beta=0.99$; symmetry $p=$ $0.179,1-\beta=0.90)$. Subject 5 's stance time increased by $7 \%$ on her powered prosthesis and $10 \%$ on her intact side, increasing her asymmetry by $13 \%$.

With increasing levels of visual feedback (i.e. None/L1 to L3), peak propulsive force significantly increased on the prosthesis-side only (prosthesis-side $p=0.012, \eta_{p}^{2}=$ 0.66 ; intact-side $p=0.931,1-\beta=0.78$ ), thus improving peak propulsion symmetry $\left(p=0.036, \eta_{p}^{2}=0.58\right)$ (Fig. 3). L3 significantly differed from None (and L1) with $22 \%$ increase in peak propulsion and $47 \%$ improvement in propulsion symmetry with the powered prosthesis. Device effects were not significant (prosthesis-side $p=$ $0.128,1-\beta=0.56$; intact-side $p=0.709,1-\beta=0.82$; symmetry $p=0.146,1-\beta=0.81$ ). Prosthesis-side peak propulsion averaged across conditions was $31 \%$ greater with the powered versus passive prosthesis $(0.70$ to $0.91 \mathrm{~W} /$ $\mathrm{kg}$ ) and peak propulsion asymmetry was $48 \%$ less with the powered versus passive prosthesis (61 to 31\%). 
Table 3 ANOVA results with the inclusion/exclusion of each identified outlier trial

\begin{tabular}{|c|c|c|c|c|}
\hline Response Measure & Included/excluded outlier trial & $\begin{array}{l}\text { Device } \\
\text { main effect }\end{array}$ & $\begin{array}{l}\text { Feedback } \\
\text { main effect }\end{array}$ & $\begin{array}{l}\text { Interaction } \\
\text { effect }\end{array}$ \\
\hline \multirow[t]{2}{*}{ Perceived difficulty } & Included & 0.157 & $<0.001$ & 0.639 \\
\hline & Excluded & 0.300 & $<0.001$ & 0.507 \\
\hline \multirow[t]{2}{*}{ Prosthesis-side peak braking force } & Included & 0.541 & 0.272 & 0.866 \\
\hline & Excluded & 0.607 & 0.252 & 0.839 \\
\hline \multirow[t]{2}{*}{ Intact-side peak hip power } & Included & 0.864 & 0.080 & 0.997 \\
\hline & Excluded & 0.840 & 0.074 & 0.979 \\
\hline
\end{tabular}

In total, we identified 3 outlier trials in 3 response variables (i.e. perceived difficulty, prosthesis-side peak braking force, intact-side peak hip power). However, the inclusion/exclusion of these identified outlier trials did not affect the significance of our results (below), so we did not exclude any trials. Because these outlier trials were not outliers in any other response variable, the removal of identified outliers may remove valuable information from this study as a whole

Subject 5 was generally able to generate more propulsion from the powered prosthesis (and propulsion symmetry) than most other subjects (Fig. 4). Comparing L3 to None, her peak propulsion increased by $4 \%$ on the prosthesis side $(0.96$ to $1.00 \mathrm{~W} / \mathrm{kg})$ and $6 \%$ on the intact side (0.91 to $0.96 \mathrm{~W} / \mathrm{kg})$, improving her propulsion asymmetry by $40 \%$ ( -6 to $-4 \%$ ).

Peak positive hip power did not significantly change with visual feedback (prosthesis-side $p=$ $0.879,1-\beta=0.40$; intact-side $p=0.080,1-\beta=0.97$ ) or device (prosthesis-side $p=0.169,1-\beta=0.26$; intactside $p=0.864,1-\beta=0.90$ ). However, individual subject comparisons demonstrate that peak positive hip power on the prosthesis-side was significantly reduced (i.e. closer to that of the intact side) with the powered prosthesis for the same subjects that exhibited greater ipsilateral propulsion with the powered prosthesis (Fig. 4).

All feedback/device interaction effects were statistically insignificant, but subject/device interactions accounted for more than $76 \%$ of the total variance for kinetic measures (Fig. 4) and 59\% for stance time.

\section{Discussion}

To our knowledge, this study is the first to evaluate the effects of extended stance time on an amputees' prosthetic limb via visual feedback with the goal of achieving greater functional benefits with a powered knee prosthesis. Our results partially support our primary hypothesis, as subjects exhibited significantly improved stance time symmetry and propulsion symmetry with visual feedback. Our remaining hypotheses were not

Table 4 Perceived difficulty, additional temporal measures, and braking force

\begin{tabular}{|c|c|c|c|c|c|c|c|c|c|c|c|}
\hline & \multicolumn{4}{|c|}{ Prescribed Prosthesis } & \multicolumn{4}{|c|}{ Powered Prosthesis } & \multicolumn{3}{|c|}{ ANOVA Results ${ }^{*}$} \\
\hline & None & Level 1 & Level 2 & Level 3 & None & Level 1 & Level 2 & Level 3 & $\begin{array}{l}\text { Device Main } \\
\text { Effect }\end{array}$ & $\begin{array}{l}\text { Feedback Main } \\
\text { Effect }\end{array}$ & $\begin{array}{l}\text { Interaction } \\
\text { Effect }\end{array}$ \\
\hline $\begin{array}{l}\text { Target-hitting accuracy } \\
\text { (s) }\end{array}$ & - & $\begin{array}{l}0.07 \pm \\
0.05\end{array}$ & $\begin{array}{l}0.06 \pm \\
0.05\end{array}$ & $\begin{array}{l}0.06 \pm \\
0.04\end{array}$ & - & $\begin{array}{l}0.08 \pm \\
0.08\end{array}$ & $\begin{array}{l}0.07 \pm \\
0.06\end{array}$ & $\begin{array}{l}0.05 \pm \\
0.03\end{array}$ & 0.833 & 0.624 & 0.352 \\
\hline Perceived Difficulty & $1 \pm 1$ & $3 \pm 2$ & $3 \pm 1$ & $4 \pm 1$ & $2 \pm 2^{c}$ & $3 \pm 1^{b}$ & $4 \pm 1^{\mathrm{ab}}$ & $5 \pm 1^{a}$ & 0.157 & $<0.001$ & 0.639 \\
\hline \multicolumn{12}{|l|}{ Stride Time (s) } \\
\hline Prosthesis side & $\begin{array}{l}1.3 \pm \\
0.1\end{array}$ & $1.3 \pm 0.01$ & $1.4 \pm 0.1$ & $1.4 \pm 0.1$ & $\begin{array}{l}1.4 \pm \\
0.1^{b}\end{array}$ & $1.3 \pm 0.1^{b}$ & $1.4 \pm 0.1^{\mathrm{ab}}$ & $1.5 \pm 0.1^{\mathrm{a}}$ & 0.243 & 0.003 & 0.959 \\
\hline Intact side & $\begin{array}{l}1.3 \pm \\
0.1\end{array}$ & $1.3 \pm 0.01$ & $1.4 \pm 0.1$ & $1.4 \pm 0.2$ & $\begin{array}{l}1.4 \pm \\
0.1^{b}\end{array}$ & $1.3 \pm 0.1^{b}$ & $1.4 \pm 0.1^{\mathrm{ab}}$ & $1.5 \pm 0.1^{\mathrm{a}}$ & 0.183 & 0.003 & 0.973 \\
\hline \multicolumn{12}{|c|}{ Normalized Stance Time (\% stride) } \\
\hline Prosthesis side & $64 \pm 3$ & $64 \pm 3$ & $64 \pm 3$ & $64 \pm 3$ & $62 \pm 2$ & $63 \pm 2$ & $63 \pm 2$ & $63 \pm 1$ & 0.400 & 0.519 & 0.645 \\
\hline Intact side & $71 \pm 2$ & $71 \pm 1$ & $70 \pm 1$ & $68 \pm 1$ & $72 \pm 2^{a}$ & $71 \pm 2^{a}$ & $71 \pm 2^{a b}$ & $69 \pm 2^{b}$ & 0.528 & 0.005 & 0.872 \\
\hline \multicolumn{12}{|c|}{ Normalized Swing Time (\% stride) } \\
\hline Prosthesis side & $36 \pm 3$ & $36 \pm 3$ & $36 \pm 3$ & $36 \pm 3$ & $38 \pm 2$ & $37 \pm 2$ & $37 \pm 2$ & $37 \pm 1$ & 0.400 & 0.519 & 0.645 \\
\hline Intact side & $29 \pm 2$ & $29 \pm 1$ & $30 \pm 1$ & $32 \pm 1$ & $28 \pm 2^{b}$ & $29 \pm 2^{b}$ & $30 \pm 2^{a b}$ & $31 \pm 2^{a}$ & 0.405 & 0.007 & 0.749 \\
\hline \multicolumn{12}{|l|}{ Peak Braking Force (W/kg) } \\
\hline Prosthesis side & $\begin{array}{l}0.6 \pm \\
0.2\end{array}$ & $0.6 \pm 0.2$ & $0.6 \pm 0.2$ & $0.7 \pm 0.2$ & $0.7 \pm 0.2$ & $0.7 \pm 0.2$ & $0.7 \pm 0.2$ & $0.7 \pm 0.2$ & 0.541 & 0.272 & 0.866 \\
\hline Intact side & $\begin{array}{l}1.0 \pm \\
0.3\end{array}$ & $0.9 \pm 0.3$ & $1.0 \pm 0.3$ & $1.0 \pm 0.3$ & $1.2 \pm 0.2$ & $1.1 \pm 0.2$ & $1.2 \pm 0.2$ & $1.3 \pm 0.2$ & 0.397 & 0.056 & 0.732 \\
\hline
\end{tabular}

${ }^{*}$ Conditions without the same superscript letter (a-c) are significantly different with Tukey's multiple comparisons adjustment (alpha $\left.=0.05\right)$. No interaction effects were significant in this study, so all superscripts correspond to the feedback main effect and are included in only the powered prosthesis columns for simplicity 


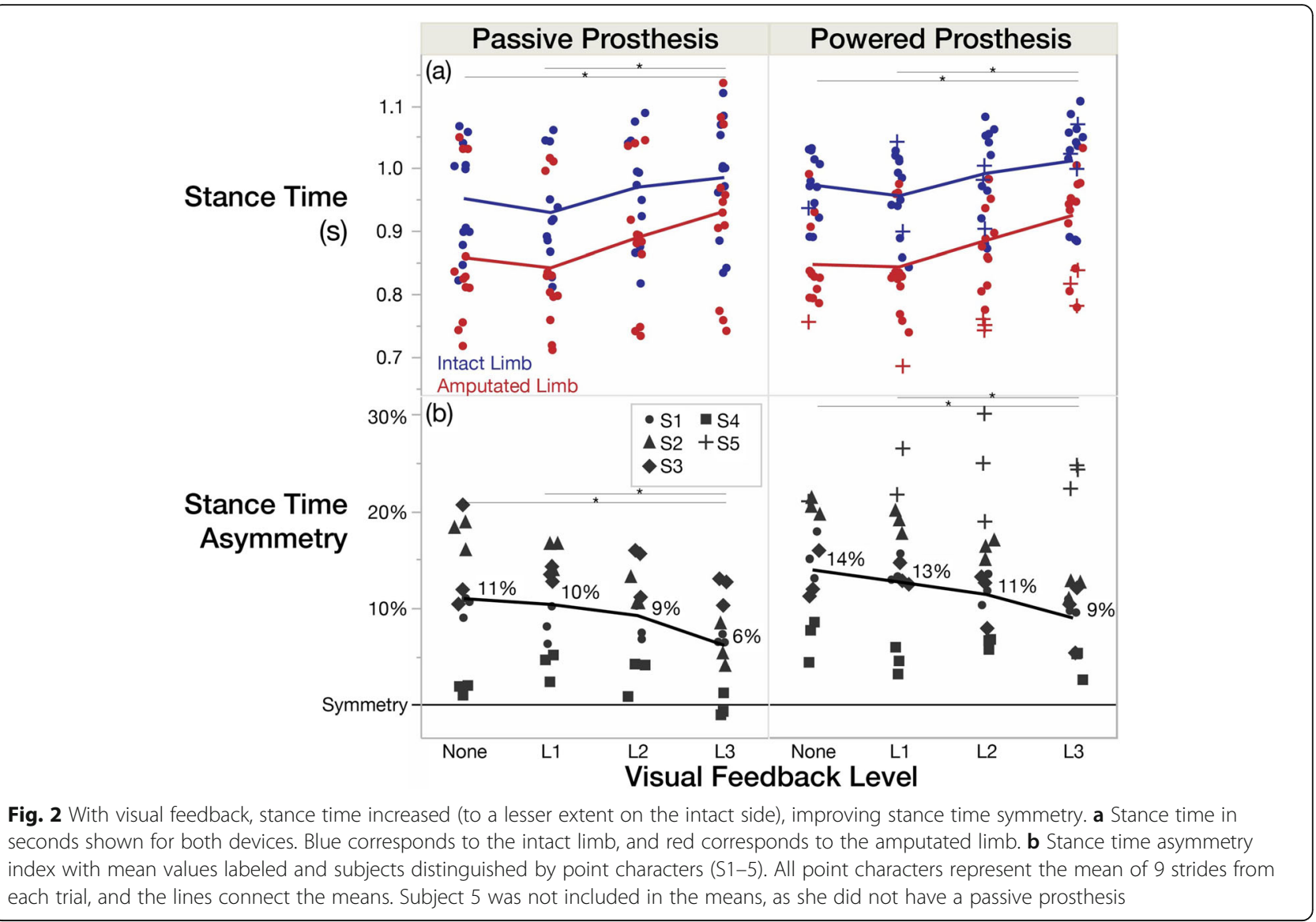

supported, as subjects responded inconsistently to the powered knee prosthesis. Given these results, we support rehabilitation and engineering approaches aimed at improving amputees' gait symmetry, but individual modifications to the device (e.g. control parameters) and/or intervention (e.g. additional physical therapist/verbal cueing) may be required.

Temporal consistency across devices is consistent with previous studies [20,29], and complete symmetry was not attained in our cohort. This lack of symmetric gait is likely due to the design of our visual feedback and combined limitations of the prosthesis (e.g. lack of a powered ankle) and/or altered musculoskeletal structure post amputation. In line with previous studies, we also observed an improvement in propulsion symmetry with stance time symmetry [30], without significantly increasing ipsilateral braking forces [31]. These results suggest that prostheses are capable of providing more assistance for amputees during locomotion if amputees use visual feedback to consciously modify their gait behavior, and heavy powered ankle components (implemented in experimental powered knee-ankle prostheses) may not be necessary to increase propulsive forces from the prosthesis.

Subjects responded differently to each prosthesis, but propulsion from the prosthetic limb was either comparable to or greater with the powered prosthesis compared to the passive prosthesis (Fig. 4). A decrease in walking speed can yield a decrease in propulsion, but Subject 3's propulsion only decreased on the intact side when switching to the powered prosthesis (and decreasing his speed), suggesting the powered prosthesis provided greater assistance than his passive prosthesis. Subject 4 (likely due to his high functional k-level) even generated greater propulsive force with the powered prosthesis compared to his intact limb, reversing his propulsion asymmetry in the opposite direction (Figs. 3 and 4). This phenomenon is quite atypical in the amputee population [7], and we believe it originates from a more posterior position of his prosthetic limb relative to his center of mass during stance phase (compared to his intact limb during stance phase). A physical therapist characterized his gait pattern as having a shortened prosthetic-side hip flexion at initial contact, a lengthened mid-stance and terminal-stance phase, and greater limb extension prior to his intact-limb's heel strike compared to other subjects. This limb extension is behavior physical therapists work hard to achieve to promote greater propulsion. Subjects may further benefit from physical therapist guidance/verbal cueing in addition to this visual feedback to achieve a 


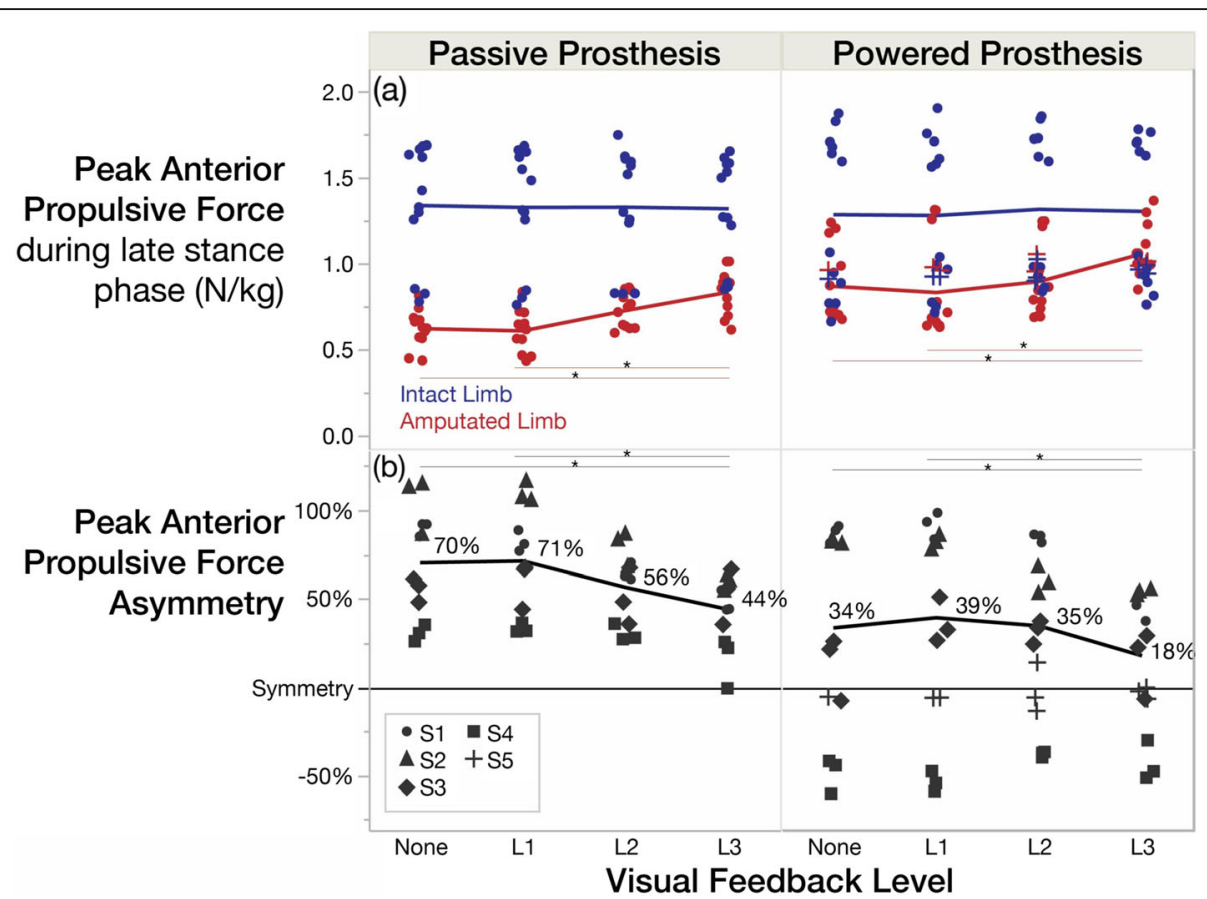

Fig. 3 With visual feedback, subjects attained greater anterior propulsion from their amputated limb with the powered prosthesis, more closely matching the propulsion of the intact limb. a Peak anterior propulsion of each limb (normalized to body weight) shown for both devices. Blue corresponds to the intact limb, and red corresponds to the amputated limb. b Peak anterior propulsion asymmetry index with mean values labeled and subjects distinguished by point characters (S1-5). All point characters represent the mean of 9 strides from each trial, and the lines connect the means. Subject 5 was not included in the means, as she did not have a passive prosthesis

more optimal posture and symmetric levels of propulsion.

For Subjects 2-4, the additional propulsion with the powered prosthesis coincided with a reduction in ipsilateral hip power, which is likely an indication of a proximal-to-distal redistribution of power [32]. Subject 3's prosthesis-side hip power reduction with the powered versus passive prosthesis may not be attributed to a decrease in speed alone, as the magnitude of power reduction was approximately twice as large as the power reduction expected in able-bodied people with the same decrease in speed [33]. Subject 4 favorably exhibited a decrease in prosthesis-side hip power with the powered prosthesis, despite an increase in speed. In a previous study, the advantages of a microprocessor knee prosthesis versus a mechanical knee prosthesis did not yield greater prosthetic-side propulsion [28], but instead, the active flexion of the powered knee prosthesis in this study may enable greater propulsion and a reduction in ipsilateral hip power for some subjects.

One of the limitations of the powered knee prosthesis is the lack of a powered ankle. The biological ankle is important for providing push-off power for symmetric gait $[32,34]$. The lack of a powered ankle might limit the level of symmetry that can be achieved by the amputees. Though, adding another powered joint distally would considerably increase the weight of the prosthesis and likely the hip power needed to swing the prosthesis. Therefore, it would be an interesting study to determine whether a powered ankle is beneficial for transfemoral amputees.

All subjects were able to modify their prosthetic-limb stance time to reach the visual feedback target provided, but we observed bilateral temporal changes with our unilaterally-targeted feedback. Intact-limb stance time also increased, but to a lesser extent than the prosthetic limb (Fig. 2), corresponding to a significant decrease in intact-limb stance time when normalized to stride time (Table 4). While off-loading the intact limb is a favorable outcome, the lack of change in prosthetic-limb stance time normalized to stride time (Table 4) suggest amputees have a reduced set of compensatory strategies and are unable to attain a significant variety of movement (particularly with the prosthesis). An increase in absolute swing time and overall stride time seemed to be necessary consequences when increasing absolute stance time with feedback. Previous studies have demonstrated unilateral propulsive biofeedback induces changes in the targeted limb only for both people with and without stroke $[35,36]$, but a prosthesis presents different challenges. Additionally, amputees may have less control over the prosthesis timing because the control 


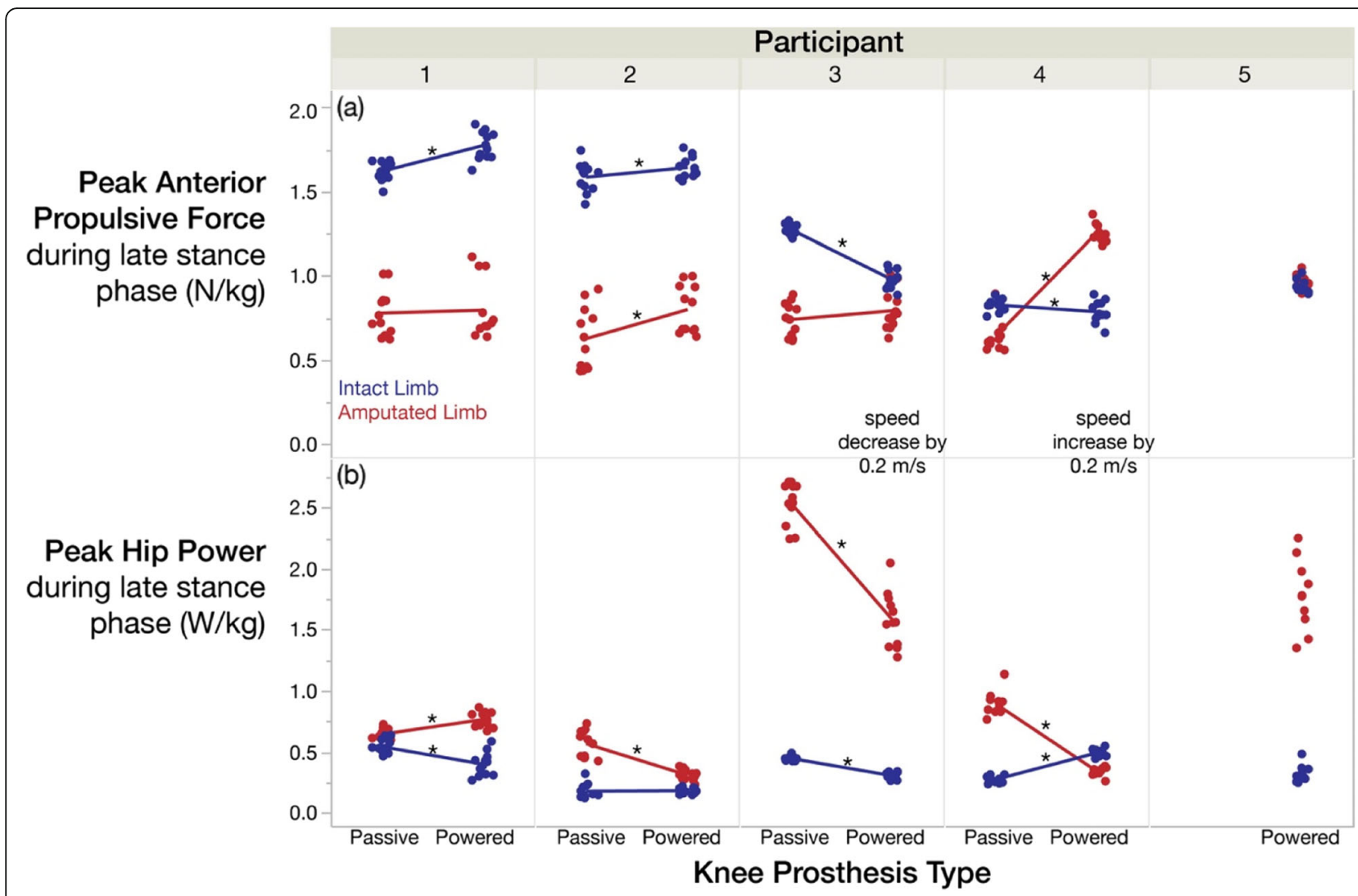

Fig. 4 Subjects responded differently to each device, but generally exhibited similar or greater propulsion with the powered knee prosthesis, coinciding with reduced ipsilateral hip power that more closely matched their intact side. a Peak anterior-posterior ground reaction force and $\mathbf{b}$ peak hip power during late stance phase, normalized to body weight. All point characters represent the mean of 9 strides from each trial, and the lines connect the means

parameters (related to swing flexion/extension timing) were fixed in this study. Physical therapy and/or adaptive powered prosthesis controllers may be necessary to increase amputees' adaptability to locomotor strategies and gait symmetry.

Interestingly, subjects' perceived difficulty increased with visual feedback level, but their target-hitting accuracy did not, suggesting they are capable of immediately walking more symmetrically, but they do not prefer it. Perceived difficulty with more symmetric gait may be associated with energetic requirements to maintain their lateral balance with longer single-support time on the prosthesis [13]. Long-term training has previously reduced transfemoral amputees' energy expenditure [37], and may reduce subjects' perceived difficulty with our visual feedback paradigm.

The improvement of both temporal and propulsive symmetry with visual feedback are very promising results for the use of this paradigm or similar therapies aimed at increasing amputees' engagement with the prosthesis. As a next step, studying the effects of longterm training and varied dosage will be useful in determining the best way to implement this paradigm in the rehabilitation process [38]. Functional benefits may further increase over time, as demonstrated in a study of transtibial amputees after 3 weeks [39]. To further increase training time, this simple visual feedback paradigm may be modified for more cost-effective equipment for use at home (e.g. pressure-sensing treadmills), or even modified to use force-sensitive resistors in insoles and auditory cues for over-ground walking (e.g. [40]), though further research is required in this direction before implementation.

On the prosthetics engineering side, this feedback paradigm may be useful in designing powered prosthesis controllers that support more symmetric gait patterns (and increased engagement with the prosthesis) rather than fitting asymmetric gait patterns learned and reinforced with passive prostheses. For example, push-off timing has been shown to be critical in optimizing amputee users' gait performance and metabolic cost [41], and researchers suggest powered knee extension timing may not be optimally timed for enhanced performance compared to passive knees [42]. The control parameters of a powered knee prosthesis have been shown to influence gait symmetry [17], but we have not yet developed 
a method of modifying the parameters to enhance gait symmetry. In our future work, we plan to develop a tuning policy for powered prostheses to maximize gait symmetry or other gait performance metrics. Moreover, the effects of maximizing user engagement with visual feedback in combination with control parameter tuning (e.g. automatic tuning $[43,44]$ ) have yet to be investigated for improved amputee gait performance.

Perhaps most importantly, inter-subject differences, common among the amputee population [19, 20, 45] and suppressed in large group averages, are important to understand for improved prescription and personalized interventions. In addition, for evaluation purposes, it may be beneficial to develop and use more standardized and consolidated metrics of symmetry [46, 47] for a more comprehensive understanding of amputees' quality of gait and comparison across studies. Note, for interventions such as visual feedback (and understanding specific behavioral changes as in this study), we support the use of targeted metrics, so the user is clear how to modify his/her gait pattern in response to the intervention.

\section{Study limitations}

We acknowledge these preliminary results reflect the behavior of a small sample size, and may not represent the general clinical population, but small sample size is common for similar studies [48-50] and allows us to take a preliminary investigation into the individual differences that may arise. Latent effects associated with testing across multiple days and limited training time may confound our comparison between devices, so we recommend an acclimation time on the order of weeks (e.g. 3 months [51]) and repeated measures with each device for a more thorough comparison. Moreover, controlling foot prosthesis type may have reduced variability, but it would not be as clinically relevant and allow optimal performance with each knee prosthesis [51]. Previous studies comparing substantially different prosthetic feet did not find significant differences in anterior-posterior ground reaction forces or stance time $[52,53]$, so we believe our interventions predominantly contributed to the results in this study. Lastly, temporal and force asymmetry may be affected by walking speed [7], but we felt it was critical to have subjects walk at a comfortable speed to ensure maximal performance with each device. The comparisons between visual feedback levels (the primary factor being studied) are within the same walking speed.

\section{Conclusion}

This study demonstrates the ability of amputee users to immediately manipulate their walking with a simple visual cue, thereby increasing their stance time and propulsive force symmetry with a commercially-available powered knee prosthesis. We believe this simple yet effective paradigm can be used as a rehabilitation tool to increase subjects' use of their prosthesis during walking, but individual user differences should be considered before implementation. Using this type of feedback in combination with more advanced prosthesis control may allow amputee users to obtain greater functional benefits from powered prostheses. We encourage research on the effects of long-term use of simple and specific biofeedback metrics for amputee rehabilitation.

\section{Abbreviations \\ L1-3: Visual feedback levels 1-3; None: No visual feedback \\ Acknowledgments \\ The authors would like to thank Derek Frankena, Stephanie Huang, Charles Humphries, David Ingraham, for their valuable time and feedback to design and conduct this study. We also thank the undergraduate researchers and all participants for their time and contributions for data collections.}

\section{Authors' contributions}

$A B, J S$, and $H H$ designed the experiment. $A B$ conducted the experiment and processed the data. All authors contributed to the analysis and interpretation of the data, and all authors read and approved the final manuscript.

\section{Funding}

This work was supported by the National Science Foundation (1563454, 1406750) and National Institute of Health (EB024570).

\section{Availability of data and materials}

The data from this study is available from the corresponding author upon reasonable request.

\section{Ethics approval and consent to participate}

All subjects provided informed, written consent to participate in our protocol approved by the Institutional Review Board of the University of North

Carolina at Chapel Hill.

\section{Consent for publication}

All subjects provided informed, written consent for publication.

\section{Competing interests}

The authors declare that they have no competing interests.

\section{Author details}

${ }^{1}$ Joint Department of Biomedical Engineering, North Carolina State University, 4402D Engineering Building III, NC State University, Raleigh, NC 27606, USA. ${ }^{2}$ The University of North Carolina at Chapel Hill, Chapel Hill, NC 27599, USA. ${ }^{3}$ Mission Gait, Richmond, VA 23228, USA. ${ }^{4}$ Department of Statistics, North Carolina State University, Raleigh, NC 27606, USA. ${ }^{5}$ Department of Allied Health Sciences, Division of Physical Therapy, The University of North Carolina at Chapel Hill, Chapel Hill, NC 27599, USA.

Received: 7 March 2019 Accepted: 28 August 2019

Published online: 11 September 2019

\section{References}

1. Burke MJ, Roman V, Wright V. Bone and joint changes in lower limb amputees. Ann Rheum Dis. 1978;37:252-4.

2. Farrokhi S, Mazzone B, Eskridge S, Shannon K, Hill OT. Incidence of overuse musculoskeletal injuries in military service members with traumatic lower limb amputation. Arch Phys Med Rehabil. 2018;99:348-54.

3. Kulkarni J, Adams J, Thomas E, Silman A. Association between amputation, arthritis and osteopenia in British male war veterans with major lower limb amputations. Clin Rehabil. 1998;12:348-53.

4. Norvell DC, Czerniecki JM, Reiber GE, Maynard C, Pecoraro JA, Weiss NS. The prevalence of knee pain and symptomatic knee osteoarthritis among 
veteran traumatic amputees and nonamputees. Arch Phys Med Rehabil. 2005;86:487-93.

5. Cutti AG, Verni G, Migliore GL, Amoresano A, Raggi M. Reference values for gait temporal and loading symmetry of lower-limb amputees can help in refocusing rehabilitation targets. J NeuroEng Rehabil. 2018;15:61.

6. Jaegers SMHJ, Arendzen $\mathrm{JH}$, de Jongh HJ. Prosthetic gait of unilateral transfemoral amputees: a kinematic study. Arch Phys Med Rehabil. 1995;76:736-43.

7. Nolan L, Wit A, Dudzinski K, Lees A, Lake M, Wychowanski M. Adjustments in gait symmetry with walking speed in trans-femoral and trans-tibial amputees. Gait Posture. 2003;17:142-51.

8. Nolan L, Lees $A$. The functional demands on the intact limb during walking for active trans-femoral and trans-tibial amputees. Prosthet Orthot Int. 2000;24:117-25.

9. Donelan JM, Kram R, Kuo AD. Mechanical work for step-to-step transitions is a major determinant of the metabolic cost of human walking. J Exp Biol. 2002;205:3717-27.

10. Penke K, Scott K, Sinskey Y, Lewek MD. Propulsive forces applied to the body's center of mass affect metabolic energetics poststroke. Arch Phys Med Rehabil. 2019;100(6):1068-75.https://doi.org/10.1016/j.apmr.2018.10.010. [Epub 2018 Nov 2]

11. Winter DA, Sienko SE. Biomechanics of below-knee amputee gait. J Biomech. 1988;21:361-7.

12. Handford ML, Srinivasan M. Energy-optimal human walking with feedbackcontrolled robotic prostheses: a computational study. IEEE Trans Neural Syst Rehabil Eng. 2018;26:1773-82.

13. Hof AL, van Bockel RM, Schoppen T, Postema K. Control of lateral balance in walking. Experimental findings in normal subjects and above-knee amputees. Gait Posture. 2007;25:250-8.

14. Liu M, Zhang F, Datseris P, Huang H. Improving finite state impedance control of active-transfemoral prosthesis using dempster-shafer based state transition rules. J Intell Robot Syst. 2013;76:461-74.

15. Ledoux ED, Goldfarb M. Control and evaluation of a powered transfemoral prosthesis for stair ascent. IEEE Trans Neural Syst Rehabil Eng. 2017;25(7): 917-24. https://doi.org/10.1109/TNSRE.2017.2656467. [Epub 2017 Jan 20]

16. Martinez-Villalpando ECSM, Herr HP. Agonist-antagonist active knee prosthesis: A preliminary study in level-ground walking. J Rehabil Res Dev. 2009:46:361-73.

17. Huang H, Crouch DL, Liu M, Sawicki GS, Wang D. A cyber expert system for auto-tuning powered prosthesis impedance control parameters. Ann Biomed Eng. 2016;44(5):1613-24.

18. Jayaraman C, Hoppe-Ludwig S, Deems-Dluhy S, McGuire M, Mummidisetty C, Siegal $R$, et al. Impact of powered knee-ankle prosthesis on low back muscle mechanics in transfemoral amputees: a case series. Front Neurosci. 2018;12:134. https:/doi.org/10.3389/fnins.2018.00134. eCollection 2018

19. Brandt A, Wen Y, Liu M, Stallings J, Huang HH. Interactions between transfemoral amputees and a powered knee prosthesis during load carriage. Sci Rep. 2017;7:14480.

20. Petersen AO, Comins J, Alkjær T. Assessment of gait symmetry in transfemoral amputees using c-leg compared with $3 R 60$ prosthetic knees. JPO. 2010;22:106-12

21. Davis BL, Ortolano M, Richards K, Redhed J, Kuznicki J, Sahgal V. Realtime visual feedback diminishes energy consumption of amputee subjects during treadmill locomotion. JPO. 2004;16:49-54.

22. Darter BJ, Wilken JM. Gait training with virtual reality-based real-time feedback: improving gait performance following transfemoral amputation. Phys Ther. 2011;91(9):1385-94. https://doi.org/10.2522/pti.20100360. [Epub 2011 Jul 14]

23. Roerdink M, Roeles S, van der Pas SC, Bosboom O, Beek PJ. Evaluating asymmetry in prosthetic gait with step-length asymmetry alone is flawed. Gait Posture. 2012;35:446-51.

24. Russell Esposito E, Choi HS, Darter BJ, Wilken JM. Can real-time visual feedback during gait retraining reduce metabolic demand for individuals with transtibial amputation? PLoS One. 2017;12.

25. Fickey SN, Browne MG, Franz JR. Biomechanical effects of augmented ankle power output during human walking. J Exp Biol. 2018;221(Pt 22):221 . https://doi.org/10.1242/jeb.182113.

26. Hanatsu N, Begg RK, Sparrow WA, Taylor S. A comparison of treadmill and overground walking effects on step cycle asymmetry in young and older individuals. J Appl Biomech. 2013;29:188-93.

27. Laferrier JZ, Gailey R. Advances in lower-limb prosthetic technology. Phys Med Rehabil Clin N Am. 2010;21:87-110.

28. Schaarschmidt M, Lipfert SW, Meier-Gratz C, Scholle H-C, Seyfarth A. Functional gait asymmetry of unilateral transfemoral amputees. Hum Mov Sci. 2012;31:907-17.
29. Kaufman KR, Frittoli S, Frigo CA. Gait asymmetry of transfemoral amputees using mechanical and microprocessor-controlled prosthetic knees. Clin Biomech (Bristol, Avon). 2012;27:460-5.

30. Dingwell JB, Davis BL, Frazier DM. Use of an instrumented treadmill for realtime gait symmetry evaluation and feedback in normal and trans-tibial amputee subjects. Prosthet Orthot Int. 1996;20:101-10.

31. Caputo JM, Collins SH. Prosthetic ankle push-off work reduces metabolic rate but not collision work in non-amputee walking. Sci Rep. 2014;4:7213.

32. Lewis CL, Ferris DP. Walking with increased ankle pushoff decreases hip muscle moments. J Biomech. 2008:41:2082-9.

33. Lelas JL, Merriman GJ, Riley PO, Kerrigan DC. Predicting peak kinematic and kinetic parameters from gait speed. Gait Posture. 2003;17:106-12.

34. Hsiao H, Knarr BA, Higginson JS, Binder-Macleod SA. The relative contribution of ankle moment and trailing limb angle to propulsive force during gait. Hum Mov Sci. 2015;39:212-21.

35. Genthe K, Schenck C, Eicholtz S, Zajac-Cox L, Wolf S, Kesar TM. Effects of real-time gait biofeedback on paretic propulsion and gait biomechanics in individuals post-stroke. Top Stroke Rehabil. 2018;25:186-93.

36. Schenck C, Kesar TM. Effects of unilateral real-time biofeedback on propulsive forces during gait. J Neuroeng Rehabil. 2017;14:52.

37. Darter BJ, Nielsen DH, Yack HJ, Janz KF. Home-based treadmill training to improve gait performance in persons with a chronic transfemoral amputation. Arch Phys Med Rehabil. 2013;94:2440-7.

38. Sawers A, Hahn ME, Kelly VE, Czerniecki JM, Kartin D. Beyond componentry: How principles of motor learning can enhance locomotor rehabilitation of individuals with lower limb loss--a review. J Rehabil Res Dev. 2012;49:1431.

39. Ray SF, Wurdeman SR, Takahashi KZ. Prosthetic energy return during walking increases after 3 weeks of adaptation to a new device. J Neuroeng Rehabil. 2018;15:6.

40. Yang L, Dyer PS, Carson RJ, Webster JB, Bo Foreman K, Bamberg SJM. Utilization of a lower extremity ambulatory feedback system to reduce gait asymmetry in transtibial amputation gait. Gait Posture. 2012;36:631-4.

41. Malcolm P, Quesada RE, Caputo JM, Collins SH. The influence of push-off timing in a robotic ankle-foot prosthesis on the energetics and mechanics of walking. J Neuroeng Rehabil. 2015;12:21.

42. Hafner BJ, Askew RL. Physical performance and self-report outcomes associated with use of passive, adaptive, and active prosthetic knees in persons with unilateral, transfemoral amputation: randomized crossover trial. J Rehabil Res Dev. 2015:52:677-700.

43. Wen Y, Si J, Gao X, Huang S, Huang H. A new powered lower limb prosthesis control framework based on adaptive dynamic programming. IEEE Trans Neural Netw Learn Syst. 2017;28(9):2215-20. https://doi.org/10. 1109/TNNLS.2016.2584559.[Epub $2016 \mathrm{Jul} 111]$.

44. Wen Y, Si J, Brandt A, Gao X, Huang H. Online reinforcement learning control for the personalization of a robotic knee prosthesis. IEEE Trans Cybern. 2019. p. 1-11. https://doi.org/10.1109/TCYB.2019.2890974. [Epub ahead of print]

45. Wentink EC, Prinsen EC, Rietman JS, Veltink PH. Comparison of muscle activity patterns of transfemoral amputees and control subjects during walking. J Neuroeng Rehabil. 2013;10:87.

46. Ramakrishnan T, Lahiff C-A, Reed KB. Comparing gait with multiple physical asymmetries using consolidated metrics. Front Neurorobot. 2018;12:2-2.

47. Torricelli D, Gonzalez-Vargas J, Veneman JF, Mombaur K, Tsagarakis N, del-Ama $\mathrm{A}$, et al. Benchmarking bipedal locomotion: a unified scheme for humanoids, wearable robots, and humans. IEEE Rob Autom Mag. 2015;22:103-15.

48. Wan AH, Wong DW, Ma CZ, Zhang M, Lee WC. Wearable vibrotactile biofeedback device allowing identification of different floor conditions for lower-limb amputees. Arch Phys Med Rehabil. 2016;97:1210-3.

49. Wolf EJ, Everding VQ, Linberg AL, Schnall BL, Czerniecki JM, Gambel JM. Assessment of transfemoral amputees using C-Leg and power knee for ascending and descending inclines and steps. J Rehabil Res Dev. 2012;49:831.

50. Buckley JG, Spence WD, Solomonidis SE. Energy cost of walking: comparison of "intelligent prosthesis" with conventional mechanism. Arch Phys Med Rehabil. 1997;78:330-3.

51. Segal AD, Orendurff MS, Klute GK, McDowell ML, Pecoraro JA, Shofer J, et al. Kinematic and kinetic comparisons of transfemoral amputee gait using CLeg and Mauch SNS prosthetic knees. J Rehabil Res Dev. 2006;43:857.

52. Zmitrewicz RJ, Neptune RR, Walden JG, Rogers WE, Bosker GW. The effect of foot and ankle prosthetic components on braking and propulsive impulses during transtibial amputee gait. Arch Phys Med Rehabil. 2006;87:1334-9. 
53. Graham LE, Datta D, Heller B, Howitt J, Pros D. A comparative study of conventional and energy-storing prosthetic feet in high-functioning transfemoral amputees. Arch Phys Med Rehabil. 2007;88:801-6.

\section{Publisher's Note}

Springer Nature remains neutral with regard to jurisdictional claims in published maps and institutional affiliations.

Ready to submit your research? Choose BMC and benefit from:

- fast, convenient online submission

- thorough peer review by experienced researchers in your field

- rapid publication on acceptance

- support for research data, including large and complex data types

- gold Open Access which fosters wider collaboration and increased citations

- maximum visibility for your research: over $100 \mathrm{M}$ website views per year

At BMC, research is always in progress. 